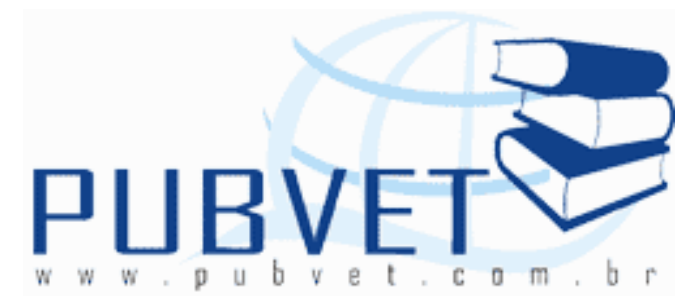

PUBVET, Publicações em Medicina Veterinária e Zootecnia.

\title{
Fisiologia reprodutiva de ovinos
}

Ernesto Lobato ${ }^{1}$, Rafael Alves da Costa Ferro ${ }^{2}$, Klayto José Gonçalves dos Santos $^{3}$, Miliane Alves da Costa, Diogo Alves da Costa Ferro, Aracele Pinheiro do Pales Santos

1 Zootecnista pela UEG - UnU de São Luís de Montes Belos

2 Docentes da UEG-UnU São Luís de Montes Belos

3 Aluna Bolsista PIBC-UEG, UnU de São Luís de Montes Belos

\section{Resumo}

A ovinocultura de corte é uma atividade que vem crescendo e se desenvolvendo muito nos últimos anos no Brasil, principalmente em regiões onde antes esta atividade era insignificante, tornando-se mais uma alternativa de investimento no meio agropecuário. O aumento da demanda por carne ovina pôs em evidência a importância do conhecimento da fisiologia reprodutiva desses animais para que possa ser empregado metodos para melhor os seus indices reprodutivos. No presente trabalho, objetivou-se abordar de forma prática e simplificada sobre a fisiologia da reprodução ovina. A fêmea ovina atinge a puberdade quando ocorre o aparecimento do primeiro estro e, o macho, quando ocorre o aparecimento de instintos reprodutivos. Os ovinos são poliéstricos estacionais e seu ciclo reprodutivo tem em torno de 17 dias, sendo dividido em proestro, estro, metaestro e diestro. O período médio de gestação é de 150 dias, com um intervalo aproximado de oito meses entre os partos. A produção de ovinos 
LOBATO, E. et al. Fisiologia reprodutiva de ovinos. PUBVET, Londrina, V. 7, N. 15, Ed. 238, Art. 1573, Agosto, 2013.

tem se mostrado uma alternativa viável no agronegócio da do país, considerando a sua eficiência produtiva e reprodutiva.

Palavras-chave: puberdade, produção, reprodução.

\title{
Reproductive physiology of sheep
}

\begin{abstract}
The sheep industry is cutting an activity that is growing and developing in recent years in Brazil, especially in regions where this activity was negligible before, becoming an alternative investment amid farming. The increased demand for lamb has highlighted the importance of knowledge of reproductive physiology of these animals so that methods can be employed to better their reproductive indices. The present study aimed to address in a practical and simplified on the physiology of reproduction in sheep. The female sheep reaches puberty occurs when the onset of the first estrus, and the male, occurs when the onset of reproductive instincts. Sheep are seasonal and poliéstricos their reproductive cycle is about 17 days, divided into proestrus, estrus, metestrus and diestrus. The average gestation period is 150 days, with an interval of approximately eight months between births. The sheep production has proved a viable alternative in the agribusiness of the country, considering their productive and reproductive efficiency.
\end{abstract}

Keywords: puberty, production, reproduction.

\section{INTRODUÇÃO}

A atividade de produção da carne ovina no Brasil tem se desenvolvido de forma gradativa, modificando o crescendo em regiões onde antes esta atividade era insignificante, tornando-a viável e também como uma nova opção de investimento no meio agropecuário, despertando assim a atenção de muitos produtores e profissionais da área. 
LOBATO, E. et al. Fisiologia reprodutiva de ovinos. PUBVET, Londrina, V. 7, N. 15, Ed. 238, Art. 1573, Agosto, 2013.

Nos últimos anos a produção e consumo da carne ovina tem tido um aumento significativo, porem apesar desse crescimento nos últimos anos, o consumo nacional é de 700 gramas por habitante por ano, sendo este considerado baixo quando comparado ao consumo na Argentina que corresponde a $1,4 \mathrm{~kg}$ e a Austrália e Nova Zelândia com 20,2 kg e 42,2 kg por habitante por ano, respectivamente (ALENCAR \& ROSA, 2006).

A produtividade de um rebanho está na dependência dos fatores que afetam sua capacidade reprodutiva. Para-se obter resultados satisfatórios na produção animal é de suma importância o conhecimento da fisiologia reprodutiva dos animais visando à otimização do manejo de rebanho quanto ao acasalamento, nascimento, desmame e abate, de forma que todas as etapas possam ser monitoradas, inclusive em relação ao planejamento nutricional e comercial.

A deficiência do setor de produção de ovinos está inteiramente correlacionada com a deficiência reprodutiva dos animais como, idade a puberdade dos animais, estacionalidade reprodutiva, detecção do cio, taxa de mortalidade e taxa de natalidade.

À medida que a eficiência reprodutiva é melhorada através de um aumento na taxa de natalidade e uma redução no índice de mortalidade dos rebanhos, tem-se um aumento no número de fêmeas para seleção, maior número de cordeiros para abate associado a um incremento na produção de carne, pele e/ou lã (MACEDO, 1993).

Objetivou-se abordar de forma prática e simplificada, a fisiologia reprodutiva dos ovinos, como, fatores que interferem na puberdade, estacionalidade reprodutiva e ciclo estral dos ovinos. 
LOBATO, E. et al. Fisiologia reprodutiva de ovinos. PUBVET, Londrina, V. 7, N. 15, Ed. 238, Art. 1573, Agosto, 2013.

\section{REVISÃO DE LITERATURA}

\subsection{Fisiologia Reprodutiva dos Ovinos}

O aparecimento da puberdade determina o início da atividade sexual tanto no macho quanto na fêmea. As fêmeas atingem a puberdade quando ocorre o aparecimento do primeiro estro. No caso dos machos, o início da atividade sexual é marcado pela presença de instintos reprodutivos e produção espermática, mesmo antes de atingirem a puberdade total, que somente será alcançada quando os espermatozóides se encontrarem viáveis para fecundação (GRANADOS et al., 2006).

De acordo com JÚNIOR \& GIRÃO (2003), os machos chegam à puberdade em torno de quatro a seis meses de idade, com o peso vivo de 22 a $28 \mathrm{~kg}$ e as fêmeas atingem a puberdade em torno de sete a dez meses de idade e, segundo BORGES \& GONÇALVES (2002), com 40 a $45 \mathrm{~kg}$ do peso adulto.

Segundo CRUZ \& FERRAZ (2012), nessa fase inicial, os animais apresentam ainda limitações reprodutivas, as fêmeas apresentam um porte anatômico inadequado à gestação e os machos apresentam um número exagerado de células espermáticas inviáveis para fertilização.

O aproveitamento prematuro da função sexual causa prejuízo no desenvolvimento normal dos animais, especialmente no caso das borregas se tornarem prenhes muito cedo (MACHADO, 2007).

Conforme QUEIROZ \& CHAVES (2010), a separação por sexos deve ser levada em consideração para que possam ser evitadas montas não programadas. Os indivíduos devem ser separados por sexo a uma idade inferior a quatro meses, período em que os machos já podem atingir a puberdade. 
LOBATO, E. et al. Fisiologia reprodutiva de ovinos. PUBVET, Londrina, V. 7, N. 15, Ed. 238, Art. 1573, Agosto, 2013.

\subsubsection{Fatores que interferem na puberdade}

Vários fatores influenciam o desencadeamento da puberdade em ovinos, entre eles podemos citar a nutrição, genética, estação de nascimento e interações sociais (MONTEIRO et al., 2010). Segundo GRANADOS et al. (2006), fatores internos como hormônios atuantes, raça (genética) e desenvolvimento ponderal também interferem no desencadeamento da puberdade. A atividade reprodutiva é comandada por processos fisiológicos, através das interações internas e externas.

$\mathrm{O}$ conhecimento desses fatores possibilita a antecipação da entrada à puberdade de fêmeas e machos e consequentemente maior rentabilidade na cadeia produtiva de ovinos (MONTEIRO et al., 2010).

\subsubsection{Estacionalidade reprodutiva}

A espécie ovina (Ovis aries) em geral é considerada como poliéstrica estacional, ou seja, os acasalamentos ocorrem de forma cíclica em uma determinada estação do ano. De fato, a ovelha apresenta estro (cio) em média, a cada 17 dias e no período do ano em que há o decréscimo no número de horas de luz por dia (fotoperíodo), ou seja, desde o final do verão e outono adentro, Figura 1 (MACHADO, 2007).

De acordo com COSTA (2007), a luminosidade é captada pelos fotorreceptores dos olhos e transmitida pelo sistema nervoso para o hipotálamo e depois para a hipófise, convertendo-se de um sinal nervoso para um sinal hormonal. Este sinal desencadeará a secreção de melatonina que irá estimular o pulso gerador do hormônio liberador de gonadotrofina (GnRH) que por sua vez irá estimular a secreção e liberação do hormônio luteinizante (LH), estimulando assim, a ovulação. Tal secreção é aumentada durante o período escuro, ou seja, em dias curtos haverá um período mais longo de alta secreção de melatonina a cada 24 horas. 
LOBATO, E. et al. Fisiologia reprodutiva de ovinos. PUBVET, Londrina, V. 7, N. 15, Ed. 238, Art. 1573, Agosto, 2013.

No Brasil, cuja área geográfica estende-se tanto sobre a linha do Equador (Regiões Nordeste e Norte) como sobre uma grande variação de latitudes ao Sul (Região Central, Sudeste e Sul), a duração da estação reprodutiva de ovinos varia consideravelmente (MARTINS et al., 2008).

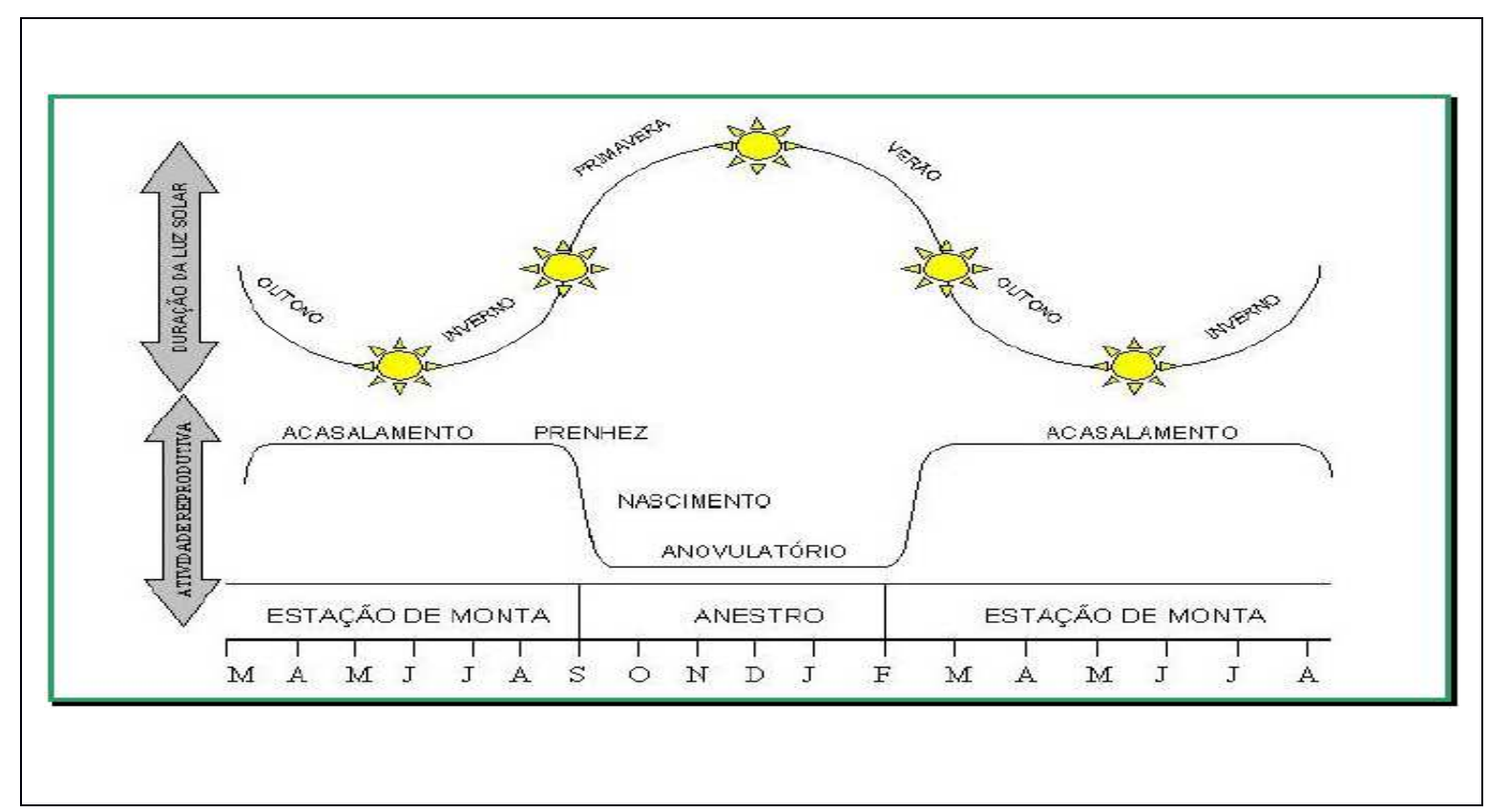

Figura 1. Esquema de estacionalidade reprodutiva em ovinos.

Fonte: Granados et al. (2006).

Segundo GRANADOS et al. (2006), de uma maneira geral os animais adaptados às condições brasileiras com o passar do tempo perdem estas características, e geralmente apresentam cio o ano todo, apenas limitado quando há carências nutricionais e sanitárias.

De acordo com FONSECA (2005), à medida que se aproxima da Linha do Equador, esta estacionalidade é diminuída. Desta maneira, em áreas subequatoriais, desde que haja aporte nutricional em quantidade e qualidade suficiente, as ovelhas ciclarão durante todo o ano. 
LOBATO, E. et al. Fisiologia reprodutiva de ovinos. PUBVET, Londrina, V. 7, N. 15, Ed. 238, Art. 1573, Agosto, 2013.

\subsubsection{Ciclo estral}

De acordo com VIU et al. (2006), o ciclo estral é um conjunto de eventos que se repetem sucessivamente, tendo a duração média de 17 dias em ovelhas. É o ritmo funcional dos órgãos reprodutivos femininos que se estabelece a partir da puberdade (GRANADOS et al., 2006).

FONSECA (2005) descreveu as fases do ciclo estral, sendo como, fase luteínica com duração de 13 dias e uma fase folicular de quatro dias. Sendo que a fase luteínica se caracteriza pela predominância da progesterona, originada do corpo lúteo, que por sua vez é formado após a ovulação e a fase folicular se caracteriza pela presença de estrógenos como hormônios principais na corrente sanguínea (QUEIROZ \& CHAVES, 2010).

De acordo com GRANADOS et al. (2006), na fase luteínica encontramse incluídos o metaestro e diestro e na fase folicular encontram-se incluídos o proestro e estro.

O conhecimento deste ciclo é de grande importância para o manejo reprodutivo porque uma vez que a fêmea tenha sido coberta, a não repetição do estro após um período de 17 dias em média, pode ser entendido como uma confirmação de gestação (CRUZ \& FERRAZ, 2012).

Os eventos que ocorrem durante $O$ ciclo estral são regulados basicamente pela interação dos hormônios $\mathrm{GnRH}$, hormônio folículo estimulante (FSH), LH, estradiol e progesterona, Quadro 1 (GRANADOS et al., 2006).

\subsubsection{Fases do ciclo estral}

\subsection{Proestro}

Fase caracterizada pelo início da produção de muco pela vulva e vagina. É o período em que a fêmea mostra-se agitada, mas ainda não aceita a monta (GRANADOS et al., 2006). 
LOBATO, E. et al. Fisiologia reprodutiva de ovinos. PUBVET, Londrina, V. 7, N. 15, Ed. 238, Art. 1573, Agosto, 2013.

Segundo OLINDA (2010), é a fase que antecede o estro (cio), onde ocorre um aumento gradativo na vascularização e tônus muscular dos órgãos genitais, relaxamento da cérvix. Tem duração média de dois a três dias e termina quando a fêmea passa a aceitar a monta do macho.

\begin{tabular}{|c|c|c|}
\hline Hormônio & Fonte & Função \\
\hline GnRH & Hipotálamo & Promove a liberação do FSH e LH \\
\hline FSH & Hipófise anterior & $\begin{array}{c}\text { Estimula o desenvolvimento folicular } \\
\text { e a secreção de estrógenos }\end{array}$ \\
\hline LH & Hipófise anterior & $\begin{array}{c}\text { Estimula a ovulação, formação e } \\
\text { manutenção do corpo lúteo }\end{array}$ \\
\hline Estradiol & Folículo (ovário) & $\begin{array}{c}\text { Estimula a manifestação do cio e a } \\
\text { liberação de LH }\end{array}$ \\
\hline Progesterona & Ovário/Placenta & Manutenção da gestação \\
\hline
\end{tabular}

Quadro 1. Principais funções dos hormônios da reprodução em fêmeas. Fonte: Granados et al. (2006).

\subsection{Estro (cio)}

Esta fase caracteriza-se pelos sinais externos de micção constantes, agitação da cauda e diminuição na ingestão de alimentos. É o período em que a fêmea deixa ser montada pelo macho (GRANADOS et al., 2006).

É de fundamental importância saber identificar corretamente a fêmea em estro para não se perder o momento certo da cobertura ou inseminação artificial (QUEIROZ \& CHAVES, 2010). Uma vez que, o estro da ovelha tem uma duração média de 30 horas, embora haja variações individuais que podem ser de 12 a 14 horas (CRUZ \& FERRAZ, 2012). 
LOBATO, E. et al. Fisiologia reprodutiva de ovinos. PUBVET, Londrina, V. 7, N. 15, Ed. 238, Art. 1573, Agosto, 2013.

\subsection{Metaestro}

De acordo com GRANADOS et al. (2006) esta é a fase onde ocorre a ovulação, com duração de 12 a 36 horas após o início do cio. Segundo OLINDA (2010), esta é a fase de difícil caracterização, pois após a ovulação por estímulo do $\mathrm{LH}$, as células da teca e da granulosa sofrem uma diferenciação em células luteínicas e formarão o corpo lúteo $(C L)$, que secretará quantidades crescentes de progesterona até atingir sua produção máxima.

\subsection{Diestro}

Nesta fase a fêmea recusa a monta e corresponde ao período em que os corpos lúteos permanecem funcionais, sendo a fase mais longa do ciclo estral, que ocorre sob predomínio da progesterona com duração variável de 17 a 18 dias. Devido à presença deste esteroide, o endométrio fica mais espesso e aumenta a atividade glandular, a cérvix se fecha, há relaxamento da musculatura genital e uma diminuição na vascularização. Esta fase termina quando ocorre a regressão fisiológica do corpo lúteo, dando inicio a um novo ciclo (LAGO \& LAFAYETTE, 2000; OLINDA, 2010).

\subsubsection{Gestação}

Segundo OLIVEIRA (2000), o período de gestação da ovelha é de aproximadamente 150 dias, o que favorece a instituição de programas de acasalamento acelerados e que objetivem obter três partos em dois anos, ou cinco partos em três anos (MACHADO, 2007).

Nos primeiros dois terços da gestação é onde ocorre a diferenciação dos órgãos e tecidos, assim como o crescimento do feto de até $30 \%$ do seu peso corporal (PILAR et al., 2002). 
LOBATO, E. et al. Fisiologia reprodutiva de ovinos. PUBVET, Londrina, V. 7, N. 15, Ed. 238, Art. 1573, Agosto, 2013.

De acordo com JúNIOR (2012), no terço final da gestação ocorre a maior parte do desenvolvimento fetal, 60 a $70 \%$ do peso da cria ao nascer, e é nessa fase que a fêmea requer maiores cuidados e, sendo um dos mais importantes à separação das fêmeas gestantes das outras e colocá-las em baias limpas, secas e bem arejadas.

Recomenda-se que ovelhas iniciem a gestação com escore de condição corporal (ECC) igual ou próximo de três pontos, em escala de um a cinco pontos, pois, a demanda nutricional aumenta de forma mais intensa em matrizes que iniciam a gestação com ECC menor que três pontos (ACCO, 2012).

\subsubsection{Intervalo de partos (IP)}

O intervalo de partos refere-se à média de dias entre dois partos consecutivos de cada animal ou de um lote. Nas ovelhas, a média é de 240 dias. O IP é um índice que depende de duas outras variáveis: o intervalo entre o parto e o primeiro estro e o número de serviços/concepção. 0 período de serviço, intervalo de tempo entre o parto e a nova concepção, varia de 66 a 90 dias, sendo este o tempo disponível para que ocorra a involução uterina, o retorno da atividade ovariana cíclica e uma nova concepção (SOUZA, 2009). Na ovelha, a involução uterina está completada aos 27 dias e precede o primeiro cio pós-parto (CERQUEIRA, 2000).

De acordo com SOUZA (2009), quanto menor o intervalo de partos maior o número de parições ocorridas dentro de um rebanho ao longo do tempo, o que consequentemente, aumentará o número de cordeiros desmamados ou produzidos anualmente.

\section{CONSIDERAÇÕES FINAIS}

A produção de ovinos tem se mostrado uma alternativa viável no agronegócio do país, considerando a sua eficiência produtiva e reprodutiva. 
LOBATO, E. et al. Fisiologia reprodutiva de ovinos. PUBVET, Londrina, V. 7, N. 15, Ed. 238, Art. 1573, Agosto, 2013.

Antes de se iniciar qualquer criação é importante fazer uma avaliação criteriosa da região, viabilidade da produção, fator climático e conhecer a fisiologia dos animais para que possa empregar técnicas que possam aperfeiçoar a produção dos mesmos visualizando a eficiência econômica da produção.

\section{REFERÊNCIAS BIBLIOGRÁFICAS}

ACCO. É possível atender a demanda nutricional de matrizes utilizando apenas a pastagem na Fase de Gestação. Online. Disponível em: http://www.acco-sc.com.br. Acesso em: 29 de outubro de 2012.

ALENCAR, Leonardo; ROSA, Fabiano R. Tito. Ovinos: Panorama e mercado. Revista 0 Berro. 96.ed. Uberaba: Agropecuária Tropical. 2006.

BARBOSA, Déborah Assis. Comportamento da parição de ovelhas-Parte 1. 2008. Online. Disponível em: http://www.farmpoint.com.br. Acesso em: 16 de outubro de 2012.

BORGES, I.; GONÇALVES, L. C. Manejo reprodutivo em ovinos. In: Manual Prático de Caprino e Ovinocultura da Universidade Federal de Minas Gerais. Belo Horizonte: 2002. p.78-81.

CERQUEIRA, Valdeane Dias. Primeiro cio pós-parto das cabras e ovelhas no Nordeste. 2000. 7f. Monografia. (Graduação em Medicina Veterinária)-UFBa/BA, Salvador. 2000.

COSTA, Ricardo Lopez Dias da. Aspectos Reprodutivos das Ovelhas. Revista Pesquisa e Tecnologia. v.4, n.1, 2007.

CRUZ, Jurandir Ferreira da; FERRAZ, Rita de Cássia Nunes. Manejo reprodutivo de caprinos e ovinos. Online. Disponível em: http://www.neppa.uneb.br. Acesso em: 16 de outubro de 2012.

FONSECA, J. F. Estratégias para o controle do ciclo estral e superovulação em ovinos e caprinos. In: Congresso Brasileiro de Reprodução Animal. 16. Goiânia: 2005. Anais: Palestras. 9p.

GRANADOS, L. B. C.; DIAS, A. J. B.; SALES, M. P. Aspectos gerais da reprodução de caprinos e ovinos. In: Capacitação dos técnicos e produtores do Norte e Noroeste Fluminense em Reprodução de Caprinos e Ovinos. 1.ed. Campos dos Goyatacazes: 2006. $54 \mathrm{p}$.

JÚNIOR, Antônio de Souza; GIRÃO, Raimundo Nonato. Manejo das crias de caprinos e ovinos. ed. Sebrae. Teresina: Plug Propaganda. 2003. 36 p. Aprisco, 1.

JÚNIOR, Edilson Soares Lopes. Manejo reprodutivo de caprinos e ovinos. Online. Disponível em: http://www.sheepembryo.com.br. Acesso em: 22 de outubro de 2012.

LAGO, Gilberto; LAFAYETTE, José Wellington de S. Manejo reprodutivo de caprinos e ovinos. 2000. Online. Disponível em: http://www.serratalhada.net. Acesso em: 18 de outubro de 2012. 
MACÊDO, F. A. R. Alguns Fatores que Afetam o Comportamento Reprodutivo e Produtivo de Ovinos da Raça Morada Nova, Variedade Vermelha. Dissertação de Mestrado) Pósgraduação em Zootecnia - Universidade Federal de Santa Maria, Santa Maria - RS, 1983. $91 \mathrm{p}$.

MACHADO, Rui. Ovinocultura: Controle da verminose, mineralização, reprodução e cruzamentos na Embrapa Pecuária Sudeste. 1. ed. São Carlos: 2007. p.28-38.

MARTINS, Charles Ferreira et al. Importância da libido na identificação de carneiros nativos de Mato Grosso do Sul, Brasil, de alta performance sexual (ABNS). In: $45^{\circ}$ Reunião Anual da Sociedade Brasileira de Zootecnia. Lavras: UFLA. 2008.

MONTEIRO, Claudia Dias; BICUDO, Sony Dimas; TOMA, Hugo Shisei. Puberdade em fêmeas ovinas. 2010. Online. Disponível em: http://www.pubvet.com.br. Acesso em: 30 de outubro de 2012.

OLINDA, Robério Gomes. Manejo reprodutivo de ruminantes. 2010. Online. Disponível em: http://www.ufersa.edu.br. Acesso em: 20 de outubro de 2012.

OLIVEIRA, Maria do Carmo P. de. Indução de parto em caprino e ovino. 2000. $12 \mathrm{f}$. Monografia. (Graduação em Medicina Veterinária)-UFBa/BA, Salvador. 2000.

PILAR, Rui de Castro; PÉREZ, Juan Rámon O.; SANTOS, Cristiane Leal dos. Manejo Reprodutivo da Ovelha: Recomendações para uma parição a cada 8 meses. In: Boletim Agropecuário da Universidade Federal de Lavras. 50.ed. Lavras: UFLA, 2002. p.128.

QUEIROZ, Anna Augusta F. de; CHAVES, Hildita Siméa de A. Manejo reprodutivo de ruminantes: Manejo reprodutivo de caprinos e ovinos. 2010. Online. Disponível em: http://www.ufersa.edu.br. Acesso em: 20 de outubro de 2012.

SOUZA, Daniel de Araújo. Elevando a produtividade: Intervalo entre partos. 2009. Online. Disponível em: http://www.farmpoint.com.br. Acesso em: 30 de outubro de 2012.

VIU, Marco Antônio de Oliveira et al. Fisiologia e manejo reprodutivo de ovinos: Revisão. Revista Eletrônica Faculdade Montes Belos. São Luís de Montes Belos: v.1, n.1, p.7998,2006 